TUBERCULOSIS

\title{
Current evidence on diagnostic accuracy of commercially based nucleic acid amplification tests for the diagnosis of pulmonary tuberculosis
}

\author{
S Greco, E Girardi, A Navarra, C Saltini
}

See end of article for authors' affiliations

Correspondence to:

Dr S Greco, Dipartimento di Malattie Polmonari,

Azienda Ospedaliera San

Camillo-Forlanini, 00151

Rome, Italy; stgreco@

scamilloforlanini.rm.it

Received 26 October 2005

Accepted 18 May 2006

Published Online First

31 May 2006
Background: Even though commercial nucleic acid amplification tests (NAATs) have become the most frequently used molecular tests for laboratory diagnosis of pulmonary tuberculosis (TB), published studies report variable estimates of their diagnostic accuracy. We analysed the accuracy of commercial NAATs for the diagnosis of pulmonary TB in smear positive and smear negative respiratory samples using culture as a reference standard.

Methods: English language studies reporting data sufficient for calculating sensitivity and specificity of commercial NAATs on smear positive and/or smear negative respiratory samples were included. Metaregression was used to analyse associations with reference test quality, the prevalence of TB, sample and test type. Predictive values for different levels of pre-test probability were quantified using Bayes' approach.

Results: Sixty three journal articles published between 1995 and 2004 met the inclusion criteria. Pooled sensitivity and specificity were 0.96 and 0.85 among smear positive samples and 0.66 and 0.98 among smear negative samples. The number of culture media used as reference test, the inclusion of bronchial samples, and the TB prevalence were found to influence the reported accuracy. The test type had no effect on the diagnostic odds ratio but seemed to be correlated with sensitivity or specificity, probably via a threshold effect.

Conclusions: Commercial NAATs can be confidently used to exclude TB in patients with smear positive samples in which environmental mycobacteria infection is suspected and to confirm TB in a proportion of smear negative cases. The methodological characteristics of primary studies have a considerable effect on the reported diagnostic accuracy. n n spite of their theoretical ability to detect even a single mycobacterial cell, nucleic acid amplification tests (NAATs) are not sufficiently reliable to replace conventional diagnostic methods for pulmonary tuberculosis (TB). Both inherent test characteristics and errors in testing procedures may account for their inaccuracy. ${ }^{1}$ As for microscopy and culture, the key factor in determining NAAT false negatives is the density of mycobacteria in the specimen, since it can result in the absence of organisms in the small volumes sampled for the test. Furthermore, the presence in respiratory secretions of enzymes capable of inhibiting amplification reactions accounts for an additional $3-25 \%$ of false negative results. ${ }^{2}$ On the other hand, false positive results arise most often from contamination of negative samples with either organisms or target DNA from samples containing large numbers of mycobacteria or from amplicons contaminating the laboratory room. ${ }^{2}{ }^{3}$

To overcome these problems, automated commercial systems were developed which were made more robust by the use of standardised procedures and reagents for sample processing, amplification, and detection. These procedures, which allow different steps of the process to take place in a single sealed tube, were intended to reduce the risk of contamination. At the same time, the use of larger sample volumes or the introduction of internal amplification controls to detect inhibitors was adopted to cut down the rate of false negative results.

Notwithstanding these precautionary measures, published studies show a considerable heterogeneity in the results obtained with commercial NAATs. The US Centers for Disease Control (CDC) recommend that commercial NAATs be used with microscopy to improve diagnostic certainty (pending culture results and/or patient's response to treatment) and that clinicians should rely on their clinical judgement in the interpretation of results. According to the $\mathrm{CDC}$, the diagnosis of pulmonary $\mathrm{TB}$ can be presumed in smear positive (acid-fast bacilli $(\mathrm{AFB})+$ ) patients with a positive NAAT result and in smear negative $(\mathrm{AFB}-$ ) patients with two subsequent positive NAAT results. Environmental mycobacterial disease can be hypothesised when a negative NAAT result is obtained from an AFB+ and inhibitor-free sample while, as about $20 \%$ of TB cases can be attributed to infection by AFB - patients, ${ }^{4}$ two negative NAAT results from two separate AFB - samples are needed to exclude contagiousness. ${ }^{5}$

Two previous meta-analyses on the accuracy of NAATs for the diagnosis of pulmonary $\mathrm{TB}$, analysing mostly home grown polymerase chain reaction (PCR) based tests, found a substantial variability in both sensitivity and specificity due to the different threshold set by each investigator and to differences in study design and quality. ${ }^{67}$ To our knowledge, the diagnostic accuracy of commercial NAATs separately on both $\mathrm{AFB}+$ and $\mathrm{AFB}$ - respiratory samples has never been systematically reviewed. This study was undertaken to assess the performance of NAATs in the context of a careful analysis of the impact of the type of test as well as of the methodological and clinical characteristics of published studies on the accuracy estimates.

Abbreviations: AFB, acid-fast bacilli; DOR, diagnostic odds ratio; MTB, Mycobacterium tuberculosis; NAAT, nucleic acid amplification test; TB, tuberculosis 


\section{METHODS}

We searched Medline to I July 2005 and Embase to 1 March 2005 using a search strategy designed to identify studies evaluating the use of commercial NAATs for the diagnosis of pulmonary TB. The titles and abstracts of the identified citations were screened and the references listed in the retrieved articles were scrutinised, considering any citation that did not obviously fail the inclusion criteria.

After a preliminary analysis of a sample of articles, studies considered eligible for inclusion in our meta-analysis were those that:

(1) examined the diagnostic performance of commercial NAATs on respiratory samples $(<5 \%$ of non-respiratory samples were tolerated);

(2) used Mycobacterium tuberculosis (MTB) culture of the same sample as the reference standard for the diagnosis of pulmonary TB;

(3) reported primary data sufficient for separately calculating both sensitivity and specificity in AFB+ and/or AFBspecimens; and

(4) were written in English.

Articles were excluded from the meta-analysis for the following reasons:

(1) reporting sensitivity and specificity "revised" by means of discrepant analysis as the only study results; in the case of studies which retested the samples on the basis of discrepant analysis, only the initial "unrevised" results were considered;

(2) possible duplicate publication: when an author or research group published more than one study, the existence of overlapping study populations was ascertained by checking sample recruitment sites and/or periods or, if these were not available, contacting authors for clarification. If this was not provided, only the article reporting the largest number of samples was included;

(3) application of commercial NAATs on gastric aspirates ( $>5 \%$ of total study sample); and

(4) analysis of previous versions of commercial NAATs.

Two investigators independently evaluated studies for inclusion and abstracted relevant data. Disagreements were reconciled by consensus.

\section{Data extraction and quality assessment}

Data were abstracted using two separate data sheets, one for $\mathrm{AFB}+$ and one for $\mathrm{AFB}-$ samples. Information recorded were descriptive data (author name, journal, publication year), type of respiratory sample, prevalence of MTB culture positive samples, testing procedures for commercial NAATs, culture and AFB staining, and commercial NAAT sensitivity and specificity.

According to the established methodological standards for evaluation of diagnostic tests, ${ }^{8}$ four aspects of study quality were examined: cohort assembly (population of recruitment, method of sample selection, data collection modality), technical quality of reference test (the use of at least two different culture media was considered a more reliable reference test), blinding, and study population features (clinical/demographic characteristics, pulmonary TB severity, and other diagnoses in subjects without pulmonary TB). The original studies in which data were collected (or primary studies) were classified according to whether each characteristic was present, absent, or unknown. In five multicentre studies the participating laboratories used different AFB staining and/or culture procedures: these items were scored as unknown. Three studies included a separate description of a subgroup of patients on antituberculous therapy: these data were not included in our analysis and the studies were scored as not including patients under treatment.

\section{Statistical analysis}

All statistical analyses were separately performed for AFB+ and $\mathrm{AFB}-$ samples. For each study we classified the commercial NAAT results as true positives (TP), false negatives (FN), false positives (FP), and true negatives (TN) as determined by comparison with MTB culture results. We then calculated the true positive rate $(\mathrm{TPR}=\mathrm{TP} /$ $\mathrm{TP}+\mathrm{FN}=$ sensitivity $)$, the true negative rate $(\mathrm{TNR}=\mathrm{TN} /$ $\mathrm{FP}+\mathrm{TN}=$ specificity), their odds $($ oddsTPR $=\mathrm{TPR} / \mathrm{l}-\mathrm{TPR}$ and oddsTNR $=\mathrm{TNR} / 1-\mathrm{TNR}$ ), and the diagnostic odds ratio (DOR) - that is, the ratio of the odds of a positive NAAT result among MTB culture positive samples compared with MTB culture negative samples (DOR $=$ oddsTPR/oddsFP rate). Thus, DOR values of $>1$ indicate good test performance while DOR values of $<1$ indicate a test more frequently positive on control subjects $(\mathrm{DOR}=1$ means that the test had no discriminating ability).

The potential problems in odds calculations associated with sensitivities and/or specificities of $100 \%$ were solved by adding 0.5 to zero values. ${ }^{9}$ In articles where two or more different commercial NAATs were analysed on the same samples, both extraction of data and calculation of accuracy measures were performed by considering them as separate studies.

To delineate the impact of study characteristics on diagnostic accuracy estimates, we fitted a multivariate random effect regression model using DOR as the dependent variable and study characteristics as explanatory variables ("Metareg", Stata 8). Since each commercial NAAT fixes a well defined numerical value as the criterion for positivity, we took into account the threshold differences between studies by simply adding the test type as covariate in the regression model. ${ }^{9}$

However, clinical interpretation of DOR is not easy as the same values can relate to different combinations of sensitivity and specificity. ${ }^{10}$ The use of fixed thresholds allowed us to explore the impact of the study characteristics (including the different thresholds chosen) on sensitivity and specificity separately. We therefore constructed two further regression models using, as dependent variables, oddsTPR and oddsTNR, respectively. For all the models the dependent variables were included after logarithmic transformation.

As explanatory variables we added the clinical and methodological characteristics of the primary studies to the regression models. Since unreported items can reflect true methodological flaws or poor reporting of a methodologically sound study, we only included variables with a percentage of unreported items of $<15 \%$. As it is known that sensitivity and specificity vary with disease prevalence when an imperfect standard is used to evaluate a test, we added to the models the proportion of MTB culture positive samples (among AFB+ or AFB - samples) as a proxy of the true prevalence of pulmonary TB..$^{11}$

The within-study variance was considered by taking weights equal to the inverse of the variance of the appropriate proportions; the between-study variance was estimated using the restricted maximum likelihood estimate (REML). ${ }^{13}$

We assessed the possibility of publication bias by evaluating a funnel plot for asymmetry, Begg's adjusted rank correlation test and Egger's regression asymmetry test ("Metabias", Stata 8). Finally, we applied Bayes' theorem to assess the changes in probability of pulmonary TB determined by the use of commercial NAATs. 
A

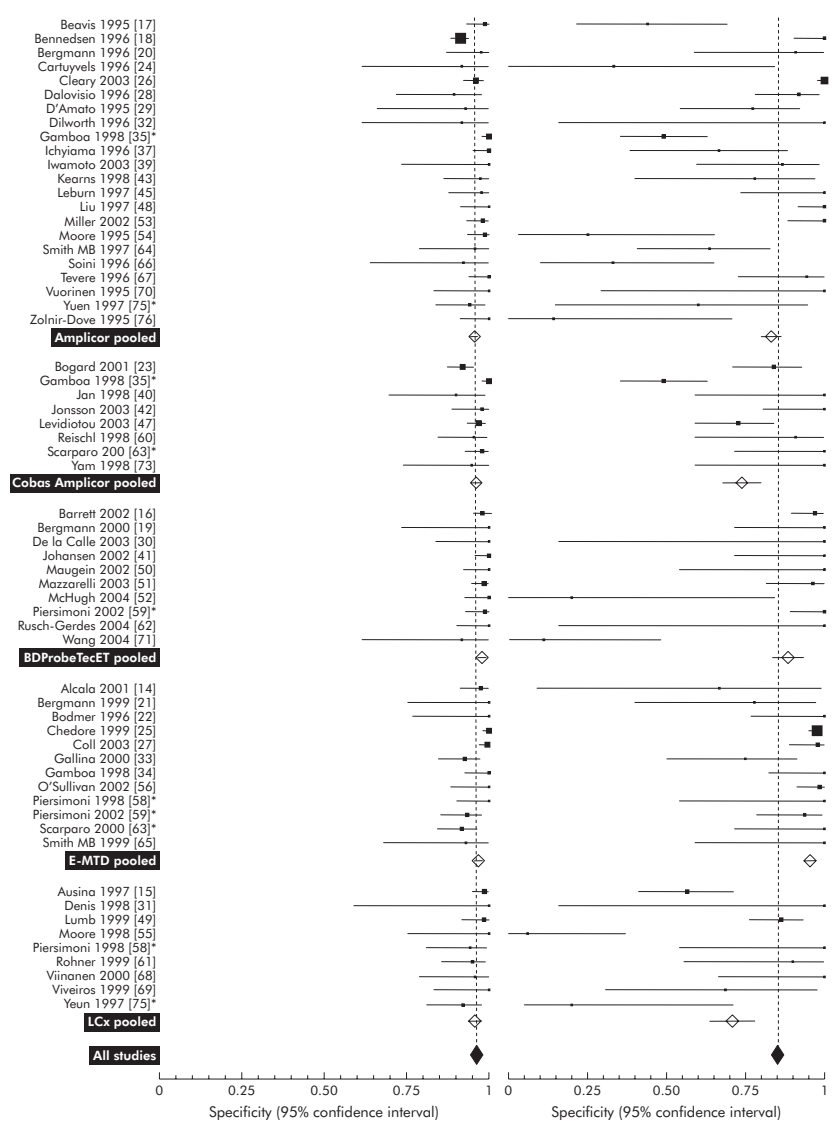

B

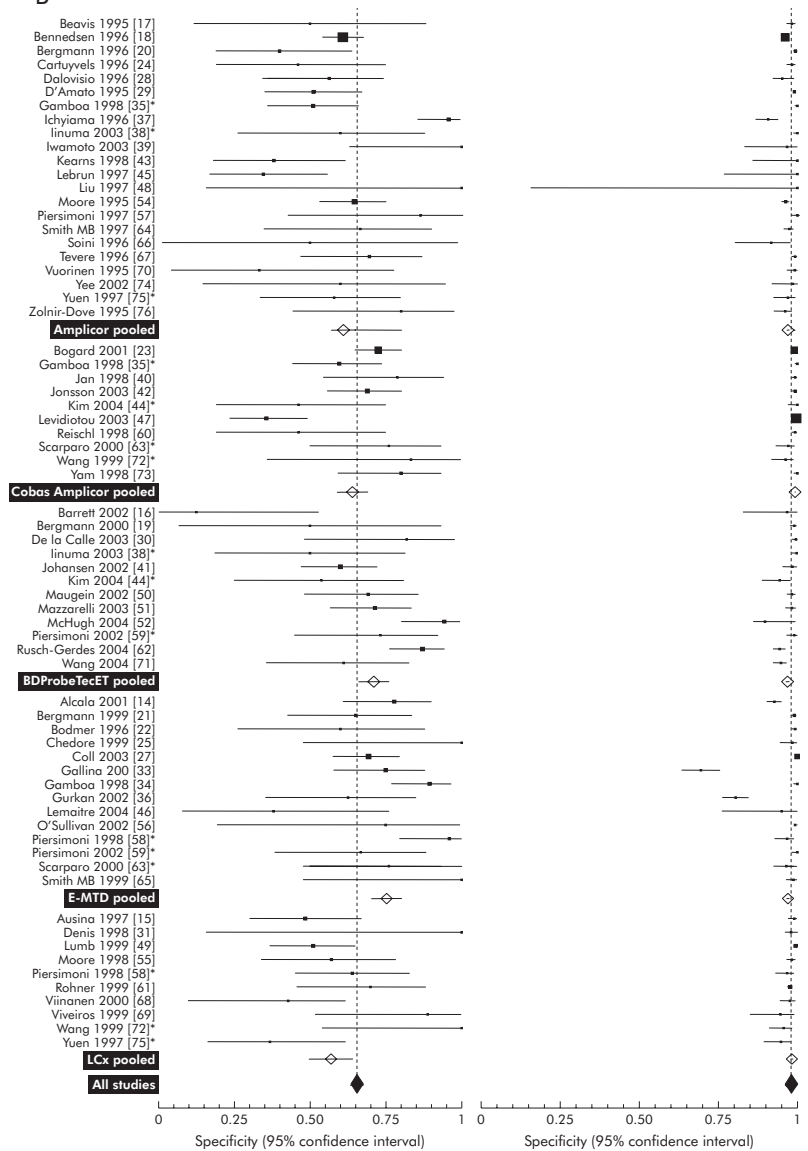

Figure 1 Individual study estimates of sensitivity and specificity of commercial nucleic acid amplification tests (NAATs) for the diagnosis of pulmonary $\mathrm{TB}$ on (A) AFB+ samples and (B) AFB - samples. Pooled values were calculated using a random effect model. Error bars represent $95 \%$ confidence intervals. Five articles on AFB+ samples and eight articles on AFB - samples analysing two commercial NAATs each are cited twice.

\section{RESULTS}

\section{Study description and synthesis}

The study selection process, which is reported in full in the online Appendix available at http://www.thoraxjnl.com/supplemental, led to the inclusion of 63 journal articles published between 1995 and 2004. ${ }^{14-76}$ Since eight articles analysed two different commercial NAATs, a total of 71 studies were available for analysis. The commercial NAATs evaluated were: Roche Amplicor MTB ( $n=25$ studies), its entirely automated version, Cobas Amplicor MTB $(n=10)$, EMTD $(n=14)$, BDProbeTecET $(n=12)$, and LCX $(n=10)$. Overall, the 63 articles examined 51160 samples, 5729 MTB culture positive and 45431 MTB culture negative. The median number of samples per study was 410 (interquartile range (IQR) 247-662), with a median pulmonary TB prevalence of 0.14 (IQR 0.07-0.3).

Fifty six articles analysed both sensitivity and specificity of commercial NAATs on AFB+ samples. They included 3848 MTB culture positive and 1535 MTB culture negative samples, with a median pulmonary TB prevalence of 0.77 (IQR 0.6-0.86). Five articles reviewed two commercial NAATs each, so 61 studies were available for analysis. As shown in fig 1A, sensitivity values were homogeneously higher $(0.96$, $95 \%$ CI 0.956 to 0.968 ) while specificity was lower and extremely variable $(0.85,95 \%$ CI 0.84 to 0.87$)$.

The 60 articles examining the sensitivity and specificity of commercial NAATs on AFB - samples included 1704 MTB culture positive and 43852 MTB culture negative samples

Table 1 Pooled values* (95\% confidence intervals) of diagnostic odds ratio (DOR), sensitivity, and specificity of five commercial nucleic acid amplification tests (NAATs)

\begin{tabular}{|c|c|c|c|c|c|c|c|}
\hline \multirow[b]{2}{*}{ Test } & \multirow{2}{*}{$\begin{array}{l}\text { NAA } \\
\text { method }\end{array}$} & \multicolumn{3}{|l|}{ AFB +} & \multicolumn{3}{|l|}{ AFB - } \\
\hline & & DOR & Sensitivity & Specificity & DOR & Sensitivity & Specificity \\
\hline Amplicor & PCR & 117 (56 to 246$)$ & $0.96(0.94$ to 0.97$)$ & $0.83(0.8$ to 0.86$)$ & $77(51$ to 115$)$ & $0.61(0.57$ to 0.65$)$ & $0.97(0.968$ to 0.974$)$ \\
\hline Cobas Amplicor & PCR & 99 (56 to 173$)$ & 0.96 (0.95 to 0.97$)$ & $0.74(0.68$ to 0.8$)$ & 220 (144 to 335$)$ & $0.64(0.59$ to 0.69$)$ & $0.993(0.992$ to 0,994$)$ \\
\hline BDP & SDA & 181 (39 to 834 ) & 0.98 (0.96 to 0.99$)$ & 0.89 (0.84 to 0.93$)$ & 96 (53 to 175$)$ & $0.71(0.66$ to 0.76$)$ & $0.97(0.964$ to 0.974$)$ \\
\hline E-MTD & TMA & 314 (99 to 995$)$ & 0.97 (0.95 to 0.98$)$ & $0.96(0.93$ to 0.97$)$ & 157 (48 to 510$)$ & $0.76(0.7$ to 0.8$)$ & $0.97(0.966$ to 0.974$)$ \\
\hline $\mathrm{LCx}$ & LCR & 42 (12 to 142$)$ & 0.96 (0.94 to 0.98$)$ & 0.71 (0.64 to 0.78$)$ & 71 (38 to 132$)$ & $0.57(0.5$ to 0.64$)$ & $0.98(0.978$ to 0.985$)$ \\
\hline
\end{tabular}

PCR, polymerase chain reaction; SDA, strand displacement amplification; TMA, trascription mediated amplification; LCR, ligase chain reaction; DOR, diagnostic odds ratio. *Random effect model. 


\begin{tabular}{|c|c|c|c|}
\hline \multirow[b]{2}{*}{ Characteristic } & \multicolumn{3}{|c|}{ Number of studies (\%) } \\
\hline & $\begin{array}{l}\text { All } \\
(n=63)\end{array}$ & $\begin{array}{l}\text { AFB+ samples } \\
(n=56)\end{array}$ & $\begin{array}{l}\text { AFB-samples } \\
(n=60)\end{array}$ \\
\hline \multicolumn{4}{|l|}{ Type of respiratory specimen } \\
\hline Sputum & $8(13)$ & $6(11)$ & $8(13)$ \\
\hline Bronchial secretions & $1(2)$ & & $1(2)$ \\
\hline Mixed respiratory secretions & $54(86)$ & $50(89)$ & $50(83)$ \\
\hline \multicolumn{4}{|l|}{ AFB method } \\
\hline Fluorescence & $45(71)$ & $43(77)$ & $42(70)$ \\
\hline Carbolfuchsin & $7(11)$ & $3(5)$ & $7(12)$ \\
\hline Unreported & $11(17)$ & $10(18)$ & $11(18)$ \\
\hline \multicolumn{4}{|l|}{ Quality of reference test } \\
\hline At least two culture media & $41(65)$ & $38(68)$ & $38(63)$ \\
\hline One culture media & $15(24)$ & $11(20)$ & $15(25)$ \\
\hline Unreported & $7(11)$ & $7(13)$ & $7(12)$ \\
\hline \multicolumn{4}{|l|}{ Commercial NAAT used } \\
\hline Amplicor & 25 & 22 & 22 \\
\hline Cobas Amplicor & 10 & 8 & 10 \\
\hline BDProbeTecET & 12 & 10 & 12 \\
\hline E-MTD & 14 & 12 & 14 \\
\hline LCx & 10 & 9 & 10 \\
\hline \multicolumn{4}{|l|}{ Population of recruitment } \\
\hline MTB culture or suspected PTB & $29(46)$ & $27(48)$ & $27(45)$ \\
\hline High PTB suspicion & $3(5)$ & $3(5)$ & $3(5)$ \\
\hline Suspected PTB or treatment monitoring & $10(16)$ & $9(16)$ & $10(17)$ \\
\hline Other & $5(8)$ & $4(7)$ & $4(7)$ \\
\hline Screening & $3(5)$ & $3(5)$ & $3(5)$ \\
\hline Unreported & $13(21)$ & $10(18)$ & $13(22)$ \\
\hline \multicolumn{4}{|l|}{ On anti-TB treatment } \\
\hline Yes & $31(49)$ & $29(52)$ & $31(52)$ \\
\hline No & $15(24)$ & $12(21)$ & $14(23)$ \\
\hline Unreported & $20(32)$ & $15(27)$ & $18(30)$ \\
\hline \multicolumn{4}{|l|}{ Method of sample selection } \\
\hline Consecutive or random selection & $20(32)$ & $17(30)$ & $20(33)$ \\
\hline Case control & $5(8)$ & $5(9)$ & $4(7)$ \\
\hline Consecutive/case control & 1 (2) & $1(2)$ & $1(2)$ \\
\hline Unreported & $37(59)$ & $33(59)$ & $35(58)$ \\
\hline \multicolumn{4}{|l|}{ Data collection modality } \\
\hline Prospective & $8(13)$ & $7(13)$ & $8(13)$ \\
\hline Retrospective & $5(8)$ & $4(7)$ & 5 (8) \\
\hline Prospective/retrospective & $2(3)$ & $2(4)$ & $2(3)$ \\
\hline Unreported & $48(76)$ & $43(77)$ & $45(75)$ \\
\hline \multicolumn{4}{|l|}{ Independence of observation } \\
\hline Any blinding & $10(16)$ & $7(13)$ & $9(15)$ \\
\hline Unreported & $53(84)$ & $49(88)$ & $51(85)$ \\
\hline \multicolumn{4}{|l|}{ Clinical/demographic characteristics } \\
\hline Reported & $3(5)$ & $3(5)$ & $3(5)$ \\
\hline Unreported & $60(95)$ & $53(95)$ & $57(95)$ \\
\hline \multicolumn{4}{|l|}{ Distribution of TB severity } \\
\hline Results of quantitative culture reported & $7(11)$ & $7(13)$ & $7(12)$ \\
\hline Unreported & $56(89)$ & $49(88)$ & $53(88)$ \\
\hline \multicolumn{4}{|l|}{ Other diagnoses in the control group } \\
\hline Reported & $1(2)$ & $1(2)$ & $1(2)$ \\
\hline Unreported & $62(98)$ & 55 (98) & $59(98)$ \\
\hline
\end{tabular}

(median pulmonary TB prevalence 0.042, IQR 0.02-0.1). Eight articles reviewed two commercial NAATs each, bringing the total number of studies up to 68. Inspection of the forest plot in fig $1 \mathrm{~B}$ indicates a high specificity but a clear heterogeneity in sensitivity values. Pooled sensitivity and specificity were 0.66 (95\% CI 0.63 to 0.68 ) and 0.98 (95\% CI 0.978 to 0.981 ), respectively. Pooled values of DOR, sensitivity, and specificity for each test type as well as their respective nucleic acid amplification techniques are reported in table 1.

Analysis of clinical and methodological characteristics of the primary studies (table 2) showed that many studies did not comply with the published guidelines for conducting and reporting diagnostic test evaluation. With regard to MTB culture (most frequently Lowenstein-Jensen (68\%) and Bactec $12 \mathrm{~B}(52 \%))$, we found that $11 \%$ of primary studies did not provide any description of the reference test used to assess pulmonary TB diagnosis, while approximately one quarter used only one culture medium. Even if more than half of the studies declared the enrollment of patients with suspected pulmonary TB, they often included samples from patients on antituberculous treatment. The clinical spectrum of both pulmonary TB and comparative groups was rarely given and only nine primary studies applied either single or double blinding for test interpretation.

\section{Effect of study characteristics on diagnostic accuracy of commercial NAATs}

The characteristics of the studies analysing AFB+ and AFBsamples are shown in the last two columns of table 2 . Those included in the meta-regression models as potential sources of heterogeneity were quality of reference test, specimen type, commercial NAAT type, and pulmonary TB prevalence. In table 3 the resulting parameter estimates of these variables are presented as relative odds. Relative odds indicate the diagnostic performance of commercial NAATs in studies with 
Table 3 Effect of study characteristics on estimates of diagnostic odds ratio (DOR), sensitivity, and specificity, as determined by meta-regression analysis

\begin{tabular}{|c|c|c|c|c|c|c|}
\hline Study characteristic & $\begin{array}{l}\text { Relative DOR } \\
(95 \% \mathrm{CI})\end{array}$ & $p$ value & $\begin{array}{l}\text { Relative oddsTPR } \\
(95 \% \mathrm{Cl})\end{array}$ & $p$ value & $\begin{array}{l}\text { Relative oddsTNR } \\
(95 \% \mathrm{CI})\end{array}$ & $p$ value \\
\hline \multicolumn{7}{|l|}{$A F B+$ samples } \\
\hline At least 2 culture media used & 8.13 (2.59 to 25.49$)$ & 0.000 & $1.64(0.90$ to 3.00$)$ & 0.106 & $2.82(1.04$ to 7.68$)$ & 0.042 \\
\hline PTB prevalence & $0.09(0.01$ to 1.72$)$ & 0.111 & $0.61(0.12$ to 3.10$)$ & 0.551 & $0.08(0.01$ to 0.96$)$ & 0.046 \\
\hline Inclusion of bronchial samples & $6.67(1.36$ to 32.63$)$ & 0.019 & $0.66(0.22$ to 1.97$)$ & 0.495 & $5.40(1.55$ to 18.79$)$ & 0.008 \\
\hline Amplicor & $0.87(0.23$ to 3.32$)$ & 0.838 & $1.08(0.56$ to 2.09$)$ & 0.812 & $0.54(0.17$ to 1.73$)$ & 0.297 \\
\hline Cobas Amplicor & 0.52 (0.11 to 2.43$)$ & 0.405 & $1.22(0.56$ to 2.6$)$ & 0.620 & $0.43(0.11$ to 1.72$)$ & 0.234 \\
\hline BDProbeTecET & $0.84(0.17$ to 4.06$)$ & 0.831 & $2.20(0.91$ to 5.30$)$ & 0.080 & $0.57(0.15$ to 2.25$)$ & 0.424 \\
\hline LCx & $0.24(0.05$ to 1.07$)$ & 0.062 & 1.06 (0.48 to 2.32$)$ & 0.893 & $0.20(0.05$ to 0.75$)$ & 0.017 \\
\hline \multicolumn{7}{|l|}{ AFB- samples } \\
\hline At least 2 culture media used & $2.26(1.13$ to 4.53$)$ & 0.021 & $0.97(0.65$ to 1.47$)$ & 0.895 & 2.77 (1.63 to 4.73$)$ & 0.000 \\
\hline PTB prevalence & $0.02(0.00$ to 0.66$)$ & 0.028 & $0.45(0.10$ to 2.16$)$ & 0.322 & $0.01(0.00$ to 0.23$)$ & 0.003 \\
\hline Inclusion of bronchial samples & $1.79(0.68$ to 4.70$)$ & 0.238 & $0.63(0.35$ to 1.16$)$ & 0.138 & $2.58(1.24$ to 5.37$)$ & 0.011 \\
\hline Amplicor & $0.91(0.36$ to 2.27$)$ & 0.839 & $0.47(0.28$ to 0.79$)$ & 0.005 & $1.90(0.93$ to 3.89$)$ & 0.081 \\
\hline Cobas Amplicor & $1.67(0.58$ to 4.82$)$ & 0.342 & $0.63(0.35$ to 1.13$)$ & 0.119 & $2.48(1.07$ to 5.76$)$ & 0.034 \\
\hline BDProbeTecET & 0.89 (0.34 to 2.32$)$ & 0.815 & $0.79(0.45$ to 1.38$)$ & 0.404 & $1.18(0.56$ to 2.48$)$ & 0.670 \\
\hline $\mathrm{LCx}$ & $0.61(0.22$ to 1.68$)$ & 0.338 & $0.44(0.24$ to 0.81$)$ & 0.008 & $1.20(0.55$ to 2.59$)$ & 0.649 \\
\hline
\end{tabular}

Results are expressed as relative odds and $95 \%$ confidence intervals.

DOR, diagnostic odds ratio; TPR, sensitivity; TNR, specificity; PTB, pulmonary tuberculosis.

The coding used in multiple regression analysis was: sample type, inclusion of bronchial specimens $=1$, sputum only $=0 ; M T B$ culture, at least two media used $=1$, no information on culture media used $=1$ (not reported in the table), one culture medium used=0; $c$ NAAT type, Amplicor $=1$, Cobas Amplicor $=1, B D P=1$,

$L C x=1, E-M T D=0$

that characteristic, relative to their performance in studies without that characteristic.

For AFB+ samples, studies using at least two MTB culture media and those including bronchial specimens yielded DOR values approximately eight times higher than those using one culture media and six times higher than those analysing sputum specimens only, mainly due to an effect on oddsTNR. OddsTNR values were inversely correlated with the prevalence of pulmonary TB and were significantly lower in studies of the LCx test than in those analysing E-MTD. For AFBsamples, the relative DOR of studies using at least two MTB culture media were more than twice as high as those of studies using only one medium, mainly due to the increase in oddsTNR. The inclusion of bronchial specimens was also associated with increased oddsTNR values. In comparison with studies analysing E-MTD, those using LCx or Roche Amplicor MTB provided lower oddsTPR, while those using Cobas Amplicor MTB yielded higher oddsTNR. An inverse correlation between the prevalence of pulmonary $\mathrm{TB}$ and both DOR and oddsTNR values was also found.

Evaluation of publication bias showed that the Egger's test was significant both for studies on AFB+ samples (regression coefficient 1.14, $\mathrm{p}=0.011$ ) and for AFB - samples (regression coefficient $0.97 \mathrm{p}=0.022$ ). Visual inspection of the two funnel plots also showed some asymmetry. Conversely, the Begg's test was not significant (see Appendix).

\section{Post-test probability of pulmonary TB}

Changes in the likelihood of pulmonary TB after performing the commercial NAATs are shown for all pre-test probabilities in fig $2 \mathrm{~A}$ and $\mathrm{B}$ for $\mathrm{AFB}+$ and $\mathrm{AFB}-$ samples. The top curves portray the positive predictive values-that is, the probabilities of pulmonary TB after obtaining a positive commercial NAAT result. The bottom curves represent the inverse of the negative predictive values-that is, the probabilities of pulmonary TB after a negative commercial NAAT result. For
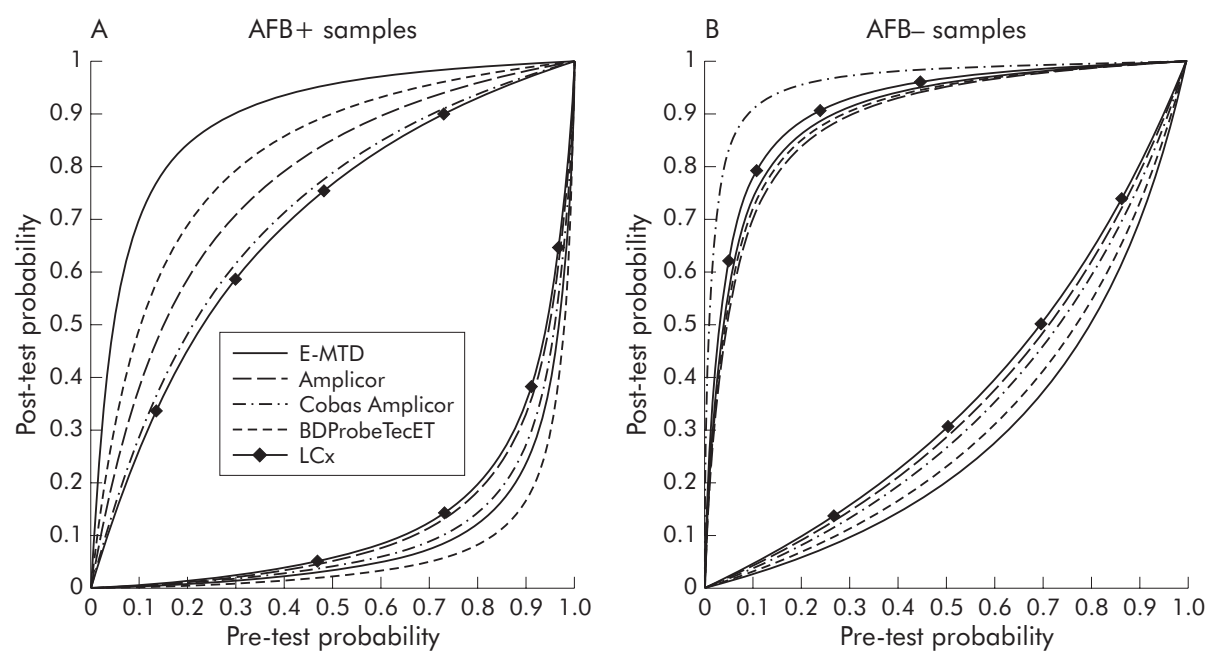

Figure 2 Predictive values of pulmonary TB after performing a commercial nucleic acid amplification test (NAAT) in (A) AFB+ samples and (B) AFBsamples. Post-test probabilities were calculated using pooled sensitivity and specificity values for each test type. Top curves represent positive predictive values, bottom curves represent 1 - negative predictive values. 
example, using E-MTD on an AFB - sample from a patient in whom previous diagnostic information (history taking, clinical examination, imaging, etc) indicated a probability of pulmonary TB of about $30 \%$, a negative result would reduce the likelihood of pulmonary TB to about $10 \%$ while a positive one would increase it to about $90 \%$.

\section{DISCUSSION}

Since commercial NAATs require fewer technical skills and a shorter assay time than the less expensive home grown tests, they have become the most frequently used molecular tests for laboratory diagnosis of pulmonary $\mathrm{TB}^{77}$ In this metaanalysis we calculated pooled estimates of their sensitivity and specificity (table 1), showed that their reported accuracy is influenced by primary study characteristics, and analysed to what degree or under what conditions they add information to the diagnostic work-up of pulmonary TB.

The reference test used for diagnosing pulmonary TB was shown to have the largest impact on accuracy, both for AFB+ and AFB - samples. Since the incorporation of one or more additional units of medium is known to reduce the false negative rates of culture, ${ }^{78}$ it could, as a consequence, have determined an "artificial" improvement in commercial NAAT specificity that is estimated on samples classified by culture as MTB-free.

The small number of studies using liquid media as the only reference test did not allow us to evaluate the independent effect on accuracy of their higher MTB recovery rates compared with solid media. ${ }^{78}$

The imperfect sensitivity of culture could also explain the variation in the specificity (and the DOR) of commercial NAATs with the prevalence of TB. At a low prevalence, the number of samples containing MTB (but wrongly classified by culture as MTB-free) is in fact likely to be small and commercial NAAT (pseudo) false positives are likely to occur less frequently. However, at a high prevalence, the higher number of (pseudo) false positives reduces the specificity. ${ }^{12}$

The higher accuracy in studies including bronchial samples, already reported in a previous meta-analysis on PCR-based NAATs, ${ }^{6}$ was mainly due to an increase in specificity. However, since the reported culture yield in bronchial samples varies from $12 \%$ to $87 \%,{ }^{79} 80$ it is difficult to explain these data on the basis of the proportional agreement of positive and negative results between the two tests. Studies focused on diagnostic performance of both culture and commercial NAATs on different bronchial samples may help to clarify this issue in the future.

Although only Amplicor (or Cobas Amplicor) and E-MTD are currently approved by the United States FDA for clinical use, the test type did not seem to explain the heterogeneity of DOR in meta-regression. Interestingly, studies evaluating EMTD on AFB - samples yielded higher sensitivities and lower specificities than those using Roche Amplicor MTB or Cobas Amplicor MTB (table 3). The higher sensitivity of E-MTD, the only NAAT approved by the FDA for use on AFB - samples, could be due to kit features such as the use of ribosomal RNA as a target sequence (about 2000 copies in each MTB cell), ${ }^{81}$ but our results suggest that E-MTD could use a lower positivity criterion than other commercial NAATs and the differences observed could be partly due to a "threshold effect". The accuracy of E-MTD appeared to be higher than that of the recently withdrawn Abbott LCx, while no differences were seen with BDProbeTecET.

With respect to the diagnostic value of commercial NAATs in the evaluation of patients with suspected pulmonary TB, we observed that, because of their very high sensitivity on $\mathrm{AFB}+$ samples, commercial NAATs can be confidently used to "rule out" pulmonary TB in AFB+ patients (fig 2A). Thus, particularly in settings where opportunistic infections are a concern, a negative inhibitor-free commercial NAAT in patients with AFB+ smears and suggestive radiographic abnormalities should direct suspicion towards an environmental mycobacterial pulmonary disease.

The more limited gain in likelihood of pulmonary TB after a positive result on $\mathrm{AFB}+$ samples (particularly for some commercial NAATs, see fig 2) seems to limit their use as confirmatory tests in these cases. The increased false positive rates of a number of studies may be related to the inclusion of samples from patients under treatment. These studies tried to correct the errors deriving from the enrollment of an inadequate study population by applying discrepant analysis, a statistical ploy that attempts to correct sensitivity and specificity of a "new" test (that is supposed to be more accurate than the reference standard it is compared with) by involving an additional more reliable test (clinical diagnosis of pulmonary TB). This procedure, by correcting the errors hidden among conflicting results of the "new" test and the standard and by disregarding concordant errors, leads to an overestimation of the accuracy of the test. ${ }^{82}$ We therefore decided to include only "uncorrected" results, hence discussing the possible effects on accuracy of the presence of samples from treated patients. The unavailability of treatment data from $27 \%$ of primary papers prevented us from drawing conclusions by means of meta-regression. Nevertheless, the pooled specificities calculated on the subgroup of studies clearly stating the exclusion $(n=206$ samples) and inclusion ( $\mathrm{n}=707$ samples) of treated patients were 0.97 (95\% CI 0.93 to 0.99 ) and 0.76 (95\% CI 0.73 to 0.79 ), respectively, indicating that inclusion of treated patient samples was the main cause for reduced specificity in AFB+ samples.

In the case of a negative microscopy result, commercial NAATs are not sensitive enough to exclude the diagnosis of pulmonary $\mathrm{TB}$ and further diagnostic work-up remains mandatory in these patients. By contrast, their high specificity gives them the ability to "rule in" pulmonary TB in about two thirds of patients who will be recognised as MTB culture positive only 2-8 weeks later (fig $2 B$ ). Based on the degree of suspicion, the clinician is allowed to initiate treatment or, having already begun it, is made more comfortable to continue it. Furthermore, and with regard to the risk assessment of infectivity, because commercial NAATs have a higher sensitivity than microscopy, they could guide the decision as to which AFB - patients are to be segregated, especially in facilities where HIV infected or other immunocompromised individuals are managed. ${ }^{83}$ However, this use is of limited value in patients already started on treatment as the numbers of viable mycobacteria in the sputum are known to dramatically fall in the first few days. ${ }^{84}$

This meta-analysis has limitations. Firstly, the sensitivity and specificity estimates of commercial NAATs are hindered by the poor quality of primary studies, a common problem in diagnostic meta-analyses. Furthermore, although the Egger's test may reveal artifactual correlations between DOR and its variance regardless of publication bias, ${ }^{85} 86$ the possibility that the studies included in our meta-analysis are a biased set cannot be ruled out. In spite of these drawbacks, we think that summary estimates of test performance are a more accurate guide for the physician than the results of any one of the primary studies.

Secondly, our decision to use the specimen as the unit of analysis could have affected accuracy because of the possible inclusion of multiple paucibacillary specimens from AFBpatients. Nevertheless, we speculated that the use of the patient as unit of analysis could have determined even wider variations in accuracy as the number of specimens per patient varied both within and between primary studies and repetitive testing is known to improve sensitivity. 
Thirdly, we examined the accuracy of commercial NAATs in comparison with culture, without addressing the issue of microscopy-negative and culture-negative pulmonary TB cases diagnosed on clinical grounds only. During the systematic review of the literature we found only six studies and one FDA premarket approval application document confronting this problem. ${ }^{81}{ }^{87-92}$ Out of 69 specimens $(52$ patients), only seven provided at least one specimen that tested positive with commercial NAATs, corresponding to a pooled sensitivity of $10 \%$ (95\% CI 4 to 20$)$. Our estimates of sensitivity on AFB - samples are therefore probably inflated compared with what can be seen in the clinical setting.

Based on this systematic review, the clinical use of commercial NAATs should be limited to the exclusion of a diagnosis of TB in AFB+ patients with suspected nontuberculous mycobacterial infection and to the confirmation of $\mathrm{TB}$ in a percentage of those providing AFB - samples. Further studies using rigorous methods-including careful control for treatment and use of single specimen per patient-would be highly desirable to appreciate better the operating characteristics of the commercial NAATs. Their accuracy on different bronchial specimens and on samples from patients with culture negative pulmonary TB are also important issues that remain to be addressed.

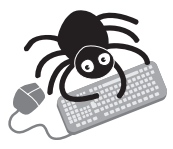

Full details of the study selection process are shown in the online Appendix available at http://www.thoraxjnl.com/supplemental.

\section{Authors' affiliations}

S Greco, Dipartimento di Malattie Polmonari, Azienda Ospedaliera San Camillo-Forlanini, Rome, Italy

S Greco, C Saltini, Dipartimento di Medicina Interna, Università di Roma "Tor Vergata", Rome, Italy

E Girardi, A Navarra, Dipartimento di Epidemiologia, INMI L Spallanzani-IRCCS, Rome, Italy

Competing interests: none declared

\section{REFERENCES}

1 Noordhoek GT, Van Embden JDA, Kolk AHJ. Questionable reliability of the polimerase chain reaction in the detection of Mycobacterium tuberculosis. N Engl J Med 1993;329:2036.

2 Richeldi L, Barnini S, Saltini C. Molecular diagnosis of tuberculosis. Eur Respir J Suppl 1995;20:689-700.

3 Noordhoek GT, Mulder S, Wallace P, et al. Multicentre quality control study for detection of Mycobacterium tuberculosis in clinical samples by nucleic amplification methods. Clin Microbiol Infect 2004;10:295-301.

4 Behr MA, Warren SA, Salomon H, et al. Transmission of Mycobacterium tuberculosis from patients smear-negative for acid-fast bacilli. Lancet 1999;353:444-9

5 Centers for Disease Control and Prevention. Nucleic acid amplification tests for tuberculosis (update). JAMA 2000;284:826.

6 Sarmiento OL, Weigle KA, Alexander J, et al. Assessment by meta-analysis of PCR for diagnosis of smear-negative pulmonary tuberculosis. J Clin Microbiol 2003;41:3233-40.

7 Flores LL, Pai M, Colford JM, et al. In-house nucleic acid amplification tests for the detection of Mycobacterium tuberculosis in sputum specimens: metaanalysis and meta-regression. BMC Microbiology 2005;5:55

8 Bossuyt P, Reitsma JB, Bruns DE, et al. The STARD statement for reporting studies of diagnostic accuracy: explanation and elaboration. Clin Chem 2003:49:7-18

9 Irwig L, Macaskill P, Glasziou P, et al. Meta-analytic methods for diagnostic test accuracy. J Clin Epidemiol 1995;48:119-30.

10 Deeks JJ. Systematic reviews of evaluations of diagnostic and screening tests. BMJ 2001;323:157-62.

11 Buck A, Gart J. Comparison of a screening test and a reference test in epidemiologic studies. Am J Epidemiol 1966:83:586-92.

12 Boyko EJ. Meta-analysis of Pap test accuracy. Am J Epidemiol 1995; 141:680-9

13 Thompson SG, Higgins JPT. How should meta-regression analyses be undertaken and interpreted? Stat Med 2002;21:1559-73.

14 Alcala L, Ruiz-Serrano MJ, Hernangomez S, et al. Evaluation of the upgraded amplified Mycobacterium tuberculosis direct test (Gen-Probe) for direct detection of Mycobacterium tuberculosis in respiratory and non-respiratory specimens. Diagn Microbiol Infect Dis 2001;41:51-6.
15 Ausina V, Gamboa F, Gazapo E, et al. Evaluation of the semiautomated AbbotLCX Mycobacterium tuberculosis assay for direct detection of Mycobacterium tuberculosis in respiratory specimens. J Clin Microbiol 1997:35:1996-2002.

16 Barrett A, Magee JG, Freeman R. An evaluation of the BDProbeTec ET system for the direct detection of Mycobacterium tuberculosis in respiratory samples. $J$ Med Microbiol 2002;51:895-8.

17 Beavis KG, Lichty MB, Jungking DL, et al. Evaluation of AMPLICOR PCR for direct detection of Mycobacterium tuberculosis from sputum specimens. J Clin Microbiol 1995;33:2582-6.

18 Bennedsen J, Thomsen VO, Pfyffer GE, et al. Utility of PCR in diagnosing pulmonary tuberculosis. J Clin Microbiol 1996;34:1407-1 1

19 Bergmann JS, Keating WE, Woods GL. Clinical evaluation of the BDProbeTec ET system for rapid detection of Mycobacterium tuberculosis. J Clin Microbiol 2000;38:863-5.

20 Bergmann JS, Woods GL. Clinical evaluation of the Roche AMPLICOR PCR Mycobacterium tuberculosis test for detection of $M$ tuberculosis in respiratory specimens. J Clin Microbiol 1996;34:1083-5.

21 Bergmann JS, Yuoh G, Fish G, et al. Clinical evaluation of the enhanced GenProbe amplified Mycobacterium tuberculosis direct test for rapid diagnosis of tuberculosis in prison inmates. J Clin Microbiol 1999:37:1419-25.

22 Bodmer T, Mockl E, Muhlemann K, et al. Improved performance of Gen-Probe amplified Mycobacterium tuberculosis direct test when 500 instead of 50 microliters of decontaminated sediment is used. J Clin Microbiol 1996;34:222-3.

23 Bogard M, Vincelette J, Antinozzi R, et al. Multicenter study of a commercial automated polymerase chain reaction system for the rapid detection of Mycobacterium tuberculosis in respiratory specimens in routine clinical practice. Eur J Clin Microbiol Infect Dis 2001;20:724-31.

24 Cartuyvels $\mathbf{R}$, De Ridder C, Jonckheere $S$, et al. Prospective clinical evaluation of Amplicor Mycobacterium tuberculosis PCR test as a screening method in a low-prevalence population. J Clin Microbiol 1996;34:2001-3.

25 Chedore $\mathbf{P}$, Jamieson FB. Routine use of the Gen-Probe MTD2 amplification test for detection of Mycobacterium tuberculosis in clinical specimens in a large public health mycobacteriology laboratory. Diagn Microbiol Infect Dis 1999;35:185-91

26 Cleary TJ, Roudel G, Casillas O, et al. Rapid and specific detection of Mycobacterium tuberculosis by using the Smart Cycler instrument and a specific fluorogenic probe. J Clin Microbiol 2003:41:4783-6.

27 Coll P, Garrigo M, Moreno C, et al. Routine use of Gen-Probe amplified Mycobacterium tuberculosis direct (MTD) test for detection of Mycobacterium tuberculosis with smear-positive and smear-negative specimens. Int J Tuberc Lung Dis 2003;7:886-91.

28 Dalovisio JR, Montenegro-James S, Kemmerly SA, et al. Comparison of the amplified M tuberculosis (MTB) direct test, Amplicor MTB PCR and IS6110PCR for detection of MTB in respiratory specimens. Clin Infect Dis 1996:23:1099-106.

29 D'Amato RF, Wallman AA, Hochstein LH, et al. Rapid diagnosis of pulmonary tuberculosis by using Roche AMPLICOR Mycobacterium tuberculosis PCR test. $J$ Clin Microbiol 1995;33:1832-4.

30 De La Calle IJ, De La Calle MAJ, Rodriguez-Iglesias M. Evaluation of the BDProbeTec ET system as screening tool in the direct detection of Mycobacterium tuberculosis complex in respiratory specimens. Diagn Microbiol Infect Dis 2003;47:573-8.

31 Denis O, Devaster JM, Vandenberg O, et al. Evaluation of ligase chain reaction for direct detection of Mycobacterium tuberculosis in respiratory specimens. Zentralbl Bakteriol 1998;288:59-65.

32 Dilworth JP, Goyal M, Young DB, et al. Comparison of polymerase chain reaction for IS61 10 and Amplicor in the diagnosis of tuberculosis. Thorax 1996:51:320-2.

33 Gallina M, Troupioti $P$, Rocco $G$, et al. Predicting culture results for Mycobacterium tuberculosis complex. Amplified Mycobacterium tuberculosis direct test and acid-fast bacilli microscopy. Chest 2000;1 18:28-32.

34 Gamboa F, Fernandez G, Padilla E, et al. Comparative evaluation of initia and new versions of the Gen-Probe amplified Mycobacterium tuberculosis direct test for direct detection of Mycobacterium tuberculosis in respiratory and nonrespiratory specimens. J Clin Microbiol 1998;36:684-9

35 Gamboa F, Manterola JM, Lonca J, et al. Comparative evaluation of two commercial assays for direct detection of Mycobacterium tuberculosis in respiratory specimens. Eur J Clin Microbiol Infect Dis 1998;17:151-7.

36 Gurkan O, Acican T, Gulbay B. Evaluation of an amplified Mycobacterium tuberculosis direct test in clinical specimens. Int J Tuberc Lung Dis 2002:6:538-41.

37 Ichiyama S, linuma Y, Tawada Y, et al. Evaluation of Gen-Probe amplified Mycobacterium tuberculosis direct test and Roche PCR-microwell plate hybridization method (Amplicor Mycobacterium) for direct detection of mycobacteria. J Clin Microbiol 1996;34:130-3.

38 linuma $Y$, Senda K, Fuijhara N, et al. Comparison of the BDProbeTec ET system with the Cobas Amplicor PCR for direct detection of Mycobacterium tuberculosis in respiratory samples. Eur J Clin Microbiol Infect Dis 2003;22:368-71

39 Iwamoto T, Sonobe T, Hayashi K. Loop-mediated isothermal amplification for direct detection of Mycobacterium tuberculosis complex, $M$ avium, and $M$ intracellulare in sputum samples. J Clin Microbiol 2003;41:2616-22.

40 Jan IS, Hsueh PR, Teng $\sqcup$, et al. Evaluation of an automatic polymerase chain reaction assay for identification of Mycobacterium tuberculosis in respiratory specimens. J Formos Med Assoc 1998;97:204-9.

41 Johansen IS, Thomsen VO, Johansen A, et al. Evaluation of a new commercial assay for diagnosis of pulmonary and nonpulmonary tuberculosis. Eur J Clin Microbiol Infect Dis 2002;21:455-60. 
42 Jonsson B, Ridell M. The Cobas Amplicor MTB test for detection of Mycobacterium tuberculosis complex from respiratory and non-respiratory clinical specimens. Scand J Infect Dis 2003;35:372-7.

43 Kearns AM, Freeman R, Steward M, et al. A rapid polymerase chain reaction technique for detecting $M$ tuberculosis in a variety of clinical specimens. J Clin Pathol 1998;51:922-4

44 Kim SY, Park YJ, Kang SJ, et al. Comparison of the BDProbeTec ET system with the Roche COBAS AMPLICOR system for detection of Mycobacterium tuberculosis complex in the respiratory and pleural fluid specimens. Diagn Microbiol Infect Dis 2004;49:13-8.

45 Lebrun L, Mathieu D, Saulnier C, et al. Limits of commercial molecular tests for diagnosis of pulmonary tuberculosis. Eur Respir J 1997;10:1874-6.

46 Lemaitre N, Armand S, Vachee A, et al. Comparison of real-time PCR method and the Gen-Probe amplified Mycobacterium tuberculosis direct test for detection of Mycobacterium tuberculosis in pulmonary and nonpulmonary specimens. J Clin Microbiol 2004;42:4307-9.

47 Levidiotou S, Vrioni G, Galanakis E, et al. Four-year experience of use of the Cobas Amplicor system for rapid detection of Mycobacterium tuberculosis complex in respiratory and nonrespiratory specimens in Greece. Eur J Clin Microbiol Infect Dis 2003;22:349-56

48 Liv D, Jones SL, Baird R, et al. Use of Roche Amplicor and multiplex PCR for diagnosis of human mycobacterial infections. Opportunistic Pathogens 1997:9:51-5.

49 Lumb R, Davies K, Dawson D, et al. Multicenter evaluation of the Abbott LCx Mycobacterium tuberculosis ligase chain reaction assay. J Clin Microbiol 1999;37:3102-7

50 Maugein J, Fourche J, Vacher S, et al. Evaluation of the BDProbeTec ET DTB assay (1) for direct detection of Mycobacterium tuberculosis complex from clinical samples. Diagn Microbiol Infect Dis 2002;44:151-5.

51 Mazzarelli G, Rindi L, Piccoli P, et al. Evaluation of the BDProbeTec ET system for direct detection of Mycobacterium tuberculosis in pulmonary and extrapulmonary samples: a multicenter study. J Clin Microbiol 2003:41:1779-82.

52 McHugh TD, Pope CF, Ling CL, et al. Prospective evaluation of BDProbeTec strand displacement amplification (SDA) system for diagnosis of tuberculosis in non-respiratory and respiratory samples. J Med Microbiol 2004;53:1215-9.

53 Miller N, Cleary T, Kraus G, et al. Rapid and specific detection of Mycobacterium tuberculosis from acid-fast bacillus smear-positive respiratory specimens and BacT/ALERT MP culture bottles by using fluorogenic probes and real-time PCR. J Clin Microbiol 2002;40:4143-7.

54 Moore DF, Curry Jl. Detection and identification of Mycobacterium tuberculosis directly from sputum sediments by AMPLICOR PCR. J Clin Microbiol 1995;33:2686-91.

55 Moore G, Curry Jl. Detection and identification of Mycobacterium tuberculosis directly from sputum sediments by ligase chain reaction. J Clin Microbiol 1998;36:1028-31.

56 O'Sullivan CE, Miller DR, Schneider PS, et al. Evaluation of Gen-Probe amplified Mycobacterium tuberculosis direct test by using respiratory and nonrespiratory specimens in a tertiary care center laboratory. J Clin Microbiol 2002;40:1723-7

57 Piersimoni C, Callegaro A, Nista D, et al. Comparative evaluation of two commercial amplification assays for direct detection of Mycobacterium tuberculosis complex in respiratory specimens. J Clin Microbiol 1997;35:193-6.

58 Piersimoni C, Callegaro A, Scarparo C et al. Comparative evaluation of the new Gen-Probe Mycobacterium tuberculosis amplified direct test and the semiautomated Abbott LCx Mycobacterium tuberculosis assay for direct detection of Mycobacterium tuberculosis complex in respiratory and extrapulmonary specimens. J Clin Microbiol 1998;36:3601-4.

59 Piersimoni C, Scarparo C, Piccoli P, et al. Performance assessment of two commercial amplification assays for direct detection of Mycobacterium tuberculosis complex from respiratory and extrapulmonary specimens. J Clin Microbiol 2002;40:4138-42.

60 Reischl U, Lehn N, Wolf $\mathrm{H}$, et al. Clinical evaluation of the automated COBAS AMPLICOR MTB assay for testing repiratory and nonrespiratory specimens. J Clin Microbiol 1998;36:2853-60.

61 Rohner $\mathrm{P}$, Jahn El, Ninet B, et al. Rapid diagnosis of pulmonary tuberculosis with the LCx Mycobacterium tuberculosis assay and comparison with conventional diagnostic techniques. J Clin Microbiol 1998;36:3046-7.

62 Rusch-Gerdes S, Richter E. Clinical evaluation of the semiautomated BDProbeTec ET system for the detection of Mycobacterium tuberculosis in respiratory and nonrespiratory specimens. Diagn Microbiol Infect Dis 2004:48:265-70

63 Scarparo C, Piccoli P, Rigon A, et al. Comparison of enhanced Mycobacterium tuberculosis amplified direct test with Cobas Amplicor Mycobacterium tuberculosis assay for direct detection of Mycobacterium tuberculosis complex in respiratory and extrapulmonary specimens. J Clin Microbiol 2000;38:1559-62.

64 Smith MB, Bergmann JS, Harris SL, et al. Evaluation of Roche Amplicor MTB assay for the detection of Mycobacterium tuberculosis in sputum specimens from prison inmates. Diagn Microbiol Infect Dis 1997;27:113-6.

65 Smith MB, Bergmann JS, Onoroto M, et al. Evaluation of the enhanced amplified Mycobacterium tuberculosis direct test for direct detection of Mycobacterium tuberculosis complex in respiratory specimens. Arch Pathol Lab Med 1999; 123:1101-3.
66 Soini H, Agha SA, El-Fiky A, et al. Comparison of amplicor and 32-kilodalton PCR for detection of Mycobacterium tuberculosis from sputum specimens. J Clin Microbiol 1996;34:1829-30.

67 Tevere VJ, Hewitt PL, Dare A, et al. Detection of Mycobacterium tuberculosis by PCR amplification with pan-Mycobacterium primers and hybridization to an M tuberculosis-specific probe. J Clin Microbiol 1996;34:918-23.

68 Viinanen AH, Soini H, Marjamaki M, et al. Ligase chain reaction assay is clinically useful in the discrimination of smear-positive pulmonary tuberculosis from atypical mycobacterioses. Ann Med 2000;32:279-83.

69 Viveiros M, Pinheiro S, Moreira P, et al. Evaluation of a commercial ligase chain reaction assay for the diagnosis of pulmonary and extra-pulmonary tuberculosis. Int J Tuberc Lung Dis 1999;3:508-14.

70 Vuorinen P, Miettinen A, Vuento R, et al. Direct detection of Mycobacterium tuberculosis complex in respiratory specimens by Gen-Probe amplified Mycobacterium tuberculosis direct test and Roche Amplicor Mycobacterium tuberculosis test. J Clin Microbiol 1995;33:1856-9.

71 Wang JY, Lee LN, Chou CS, et al. Performance assessment of a nested-PCR assay (the rapid BAP-MTB) and the BDProbeTec ET system for detection of Mycobacterium tuberculosis in clinical specimens. J Clin Microbiol 2004;43:4599-603.

72 Wang SX, Tay L. Evaluation of three nucleic acid amplification methods for direct detection of Mycobacterium tuberculosis complex in respiratory specimens. J Clin Microbiol 1999:37:1932-4.

73 Yam WC, Yuen KY, Seto WH. Direct detection of Mycobacterium tuberculosis in respiratory specimens using an automated DNA amplification assay and a single tube nested polymerase chain reaction (PCR). Clin Chem Lab Med 1998;36:597-9.

74 Yee YC, Gough A, Kumarasinghe G, et al. The pattern of utilisation and accuracy of a commercial nucleic acid amplification test for the rapid diagnosis of Mycobacterium tuberculosis in routine clinical practice. Singapore Med J 2002;43:415-20.

75 Yuen KY, Yam WC, Wong LP, et al. Comparison of two automated DNA amplification systems with a manual one-tube nested PCR assay for diagnosis of pulmonary tuberculosis. J Clin Microbiol 1997;35:1385-9.

76 Zolnir-Dovc M, Poljak M, Seme K, et al. Evaluation of two commercial amplification assays for detection of Mycobacterium tuberculosis complex in respiratory specimens. Infection 1995;23:216-21.

77 Centers for Disease Control, Prevention (CDC). M tuberculosis nucleic acid amplification testing performance evaluation program. Atlanta, CDC: 2004, http://www.phppo.cdc.gov/mpep/pdf/mtb/0401 naa.pdf (accessed 10 September 2005).

78 Pfyffer G, Welscher HM, Kissling P, et al. Comparison of the Mycobacteria Growth Indicator Tube (MGIT) with radiometric and solid culture for recovery of acid-fast bacilli. J Clin Microbiol 1997;35:364-8.

79 Kennedy DJ, Lewis WP, Barnes PF. Yield of bronchoscopy for the diagnosis of tuberculosis in patients with human immunodeficiency virus infection. Chest 1992;102:1040-4.

80 Baughman RP, Dohn MN, Loudon RG, et al. Bronchoscopy with bronchoalveolar lavage in tuberculosis and fungal infections. Chest 1991;99:92-7.

81 US Food and Drug Administration, Center for Devices and Radiological Health. Gen-Probe ${ }^{\circledR}$ Amplified ${ }^{T M}$ Mycobacterium Tuberculosis Direct (MTD) Test-P940034/S008. Part 2, pages 15-25, http://www.fda.gov/cdrh/pdf/ P940034S008b.pdf (accessed 10 September 2005).

82 Hadgu A. Discrepant analysis: a biased and an unscientific method for estimating test sensitivity and specificity. J Clin Epidemiol 1999:52:1231-7.

83 Joint Tuberculosis Committee of the British Thoracic Society. Control and prevention of tuberculosis in the United Kingdom: code of practice 2000. Thorax 2000;55:887-901.

84 Jindani A, Aber VR, Edwards EA, et al. The early bactericidal activity of drugs in patients with pulmonary tuberculosis. Am Rev Respir Dis 1980;121:939-49.

85 Deeks JJ, Macaskill P, Irwig L. The performance of tests of publication bias and other sample size effects in systematic reviews of diagnostic test accuracy was assessed. J Clin Epidemiol 2005;58:882-93.

86 Begg CB. Systematic reviews of diagnostic studies require study by study examination: first for heterogeneity and then for sources of heterogeneity. $J$ Clin Epidemiol 2005;58:865-6.

87 Jouveshomme S, Cambau E, Trystram D, et al. Clinical utility of an amplification test based on ligase chain reaction in pulmonary tuberculosis Am J Respir Crit Care Med 1998;158:1096-101.

88 Lim TK, Mukhopadhyay A, Gough A, et al. Role of clinical judgment in the application of a nucleic acid amplification test for the rapid diagnosis of pulmonary tuberculosis. Chest 2003;124:902-8.

89 Al Zahrani K, Al Jahdali $\mathrm{H}$, Poirier $\mathrm{L}$, et al. Yield of smear, culture and amplification tests from repeated sputum induction for the diagnosis of pulmonary tuberculosis. Int J Tuberc Lung Dis 2001;5:855-60.

90 Kwiatkowska S, Marczak J, Zieba M, et al. Clinical utility of a commercial ligase chain reaction kit for the diagnosis of smear-negative pulmonary tuberculosis. Int J Tuberc Lung Dis 1999;3:421-5.

91 Lim TK, Gough A, Chin NK, et al. Relationship between estimated pretest probability and accuracy of automated Mycobacterium tuberculosis assay in smear-negative pulmonary tuberculosis. Chest 2000;1 18:641-7.

92 Lim TK, Zhu D, Gough A, et al. What is the optimal approach for using a direct amplification test in the routine diagnosis of pulmonary tuberculosis? A preliminary assessment. Respirology 2002;7:351-7. 